\title{
inventio
}

La génesis de la cultura universitaria en Morelos

Año 15, núm. 37, noviembre 2019

ISSN: 2007-1760 (impreso) 2448-9026 (digital) | DOI: 10.30973/inventio/2019.15.37/6

CIENCIA Y TECNOLOGÍA

\section{Análisis químico proximal de Corydalus sp. y su comparación con insectos comestibles}

\section{América Ivette Barrera Molina}

ORCID: 0000-0003-4696-2654/ivette.bm@gmail.com

Profesora-investigadora, Facultad de Nutrición, Universidad Autónoma del Estado de Morelos (UAEM)

\section{RESUMEN}

El objetivo del presente trabajo es realizar un análisis proximal de los principales macronutrimentos presentes en el insecto $M$. Corydalus sp., mediante métodos oficiales de análisis proximales y su comparación con otros insectos comestibles. Los resultados obtenidos demostraron que este insecto cuenta con $23.18 \mathrm{~g}$ de hidratos de carbono, $17.22 \mathrm{~g}$ de grasas totales y $59.6 \mathrm{~g}$ de proteína. Los datos obtenidos demostraron un nivel proteico mayor en comparación con datos reportados para otros insectos comestibles más populares en México, como el jumil (atizies taxcuensis), la hormiga chicatana (atta mexicana), el escamol (liometopum apiculatum) y el gusano de maguey (aegiale hesperiaris). De igual forma, se observó en esta especie un aporte nutrimental superior al de la carne de bovino, ya que cuenta con $21 \mathrm{~g}$ de proteína en $100 \mathrm{~g}$ de muestra, lo cual es significativo y lo convierte en una alternativa de alimento a nuestra dieta tradicional y puede ayudar a contrarrestar los problemas de desnutrición en la población.

\section{PALABRAS CLAVE}

entomofagia; megaloptera; Corydalus sp.; análisis proximal; nutrición;

Universidad Autónoma del Estado de Morelos / Secretaría Académica

Dirección de Publicaciones y Divulgación

inventio.uaem.mx, inventio@uaem.mx 
Una de las principales problemáticas que captan la atención de la Organización de las Naciones Unidas para la Alimentación y la Agricultura (FAO) es la inseguridad alimentaria, que es la falta de acceso físico y económico de las personas a alimentos inocuos y nutritivos que cubran sus necesidades. Se ha previsto que en el año 2030 el planeta contará con nueve mil millones de personas, lo cual denota que la producción, disponibilidad y acceso a los alimentos se verán afectados y, por lo tanto, no se podrán cubrir las necesidades de la población en un futuro, a causa de la sobrepoblación, la contaminación y el mal manejo de los alimentos (Halloran \& Vantomme, 2013).

Una de las alternativas para contrarrestar el problema de alimentos con aporte nutricional que ha tenido gran aceptación en México, debido a que ya lo practicaban nuestros antepasados, es la entomofagia (del griego éntomos, insectos, y phagia, comer). Varias investigaciones sobre los insectos comestibles han demostrado que garantizan un aporte de calidad en macronutrimentos y micronutrimentos. Por mencionar algunos, están el necuazcatl (hormiga de miel), xomitl (jumil), xamoe (gusano de mezquite), pipioli (abejas), meocuilin (gusano blanco de maguey, que a su vez se compone de las voces meo, maguey, y ocuilin, gusano), azcat/molli (escamoles, de azcatt, hormiga, y molli, guisado), y chilocuilin (gusano rojo de maguey), también conocido como chinicuilomichicuil (Ramos-Elorduy, Pino M. \& Cuevas Correa, 1998).

Entre los macronutrimentos indispensables se encuentran las proteínas, que forman parte estructural y funcional en el cuerpo. Están constituidas por la combinación de sólo veinte aminoácidos, pero los seres humanos no podemos fabricar diez de ellos, por lo que deben consumirse mediante la dieta. Se dice que es un alimento de calidad aquél que contenga un número considerable de esos veinte aminoácidos. Por otro lado, la proteína animal que se consume proviene de los mamíferos, como la res, cerdo, cabra, entre otros, y sólo se encuentra en la carne, pero las proteínas que proporcionan los insectos están en todos sus componentes (Vantomme, 2010).

En cuanto a los lípidos o grasas, estos nutrientes comprenden la principal reserva energética y se recomienda su consumo, dado que el aporte calórico más recomendado proviene de grasas insaturadas; sin embargo, en algunos alimentos se encuentran presentes grasas saturadas, que son las culpables de enfermedades como la obesidad y la ateroesclerosis, por mencionar algunas. En algunos insectos comestibles se ha registrado contenido importante de grasas insaturadas, principalmente ácido oleico y ácido linolénico. Su concentración tiende a variar debido al estado en que se encuentre el insecto. En los adultos la cantidad de grasa disminuye en comparación con los estados inmaduros (Ramos Rostro, Quintero Salazar \& Ramos Elorduy, 2012).

Por otro lado, los hidratos de carbono, al igual que los lípidos, forman parte importante de la reserva energética, así como del buen funcionamiento del sistema nervioso y en la 
producción de algún aminoácido. Éstos se dividen en simples y complejos, y son los últimos los más saludables por su contenido de fibra, que promueve un desdoblamiento lento $y$, por lo tanto, estabiliza los niveles de glucosa en sangre. En los insectos se ha observado la presencia de quitina, un hidrato de carbono que podría ser considerado como fibra dietética (Kumar et al., 2004), que los convierte en una fuente muy importante de fibra, especialmente en las especies con exoesqueleto duro (Kourimsa \& Adámkova et al., 2016).

Por lo anterior, el estudio de la composición química de los insectos es de suma relevancia para la nutrición, ya que permitiría aumentar su consumo e innovar en productos que aporten los nutrientes necesarios a la población mexicana y que al mismo tiempo sean más accesibles para ella. El objetivo del presente trabajo fue realizar un análisis proximal en el insecto Corydalus sp. (Megaloptera) para conocer su calidad e incorporarlo a las tablas registradas de insectos comestibles en México.

\section{Material y métodos}

\section{Ubicación de la zona de muestreo}

El presente estudio se realizó en el río Tembembe, ubicado en la comunidad nahua de Cuentepec, municipio de Temixco, Morelos, México (figura 1), que se encuentra en las coordenadas gps latitud $18.860278^{\circ}$ y longitud $-99.326389^{\circ}$; la localidad se sitúa a una altitud de 1390 msnm. Este río se ubica en los estados de Morelos y México; sin embargo, nace en este último, en el municipio de Ocuilan, en el Sistema Volcánico Transversal, a una altitud de 3500 msnm, y desemboca en el río Chalma (figura 1) (INEGI, 2010).

\section{Descripción del megalóptero Corydalus sp.}

Los habitantes de la comunidad llaman acolotl al megalóptero Corydalus sp., designación que proviene del náhuatl atl, agua, y colotl, alacrán, es decir, alacrán de agua, de acuerdo con los habitantes del lugar. Este insecto pertenece al orden Megaloptera, cuya etimología proviene del griego, megále, grande, y pteron, ala (Contreras Ramos \& Rosas, 2014). El megalóptero Corydalus sp. es un género euritópico, es decir, ocupa un amplio espectro de condiciones ambientales en su hábitat. Pertenece a la familia Corydalidae, que es el grupo de mayor diversidad en América del sur. El género Corydalus incluye 29 especies descritas en la región, cinco de ellas registradas en México. En Morelos existen registros de tres especies, con una mayor distribución de Corydalus texanus, por lo cual puede ser ésta la especie presente en Temixco, aunque se requiere corroboración (Mayorga Villalobos, Márquez López \& Contreras Ramos, 2019). Estas especies tienen características esenciales que han logrado su supervivencia hasta nuestros días (figura 2) (Domínguez \& Fernández E., 2009).

Año 15, núm. 37, noviembre 2019

ISSN: 2007-1760 (impreso) 2448-9026 (digital) | DoI: 10.30973/inventio/2019.15.37/6 
Figura 1

Ubicación geográfica de Cuentepec en el municipio de Temixco, Morelos. Vista aérea del río Tembembe pasando por la comunidad de Cuentepec

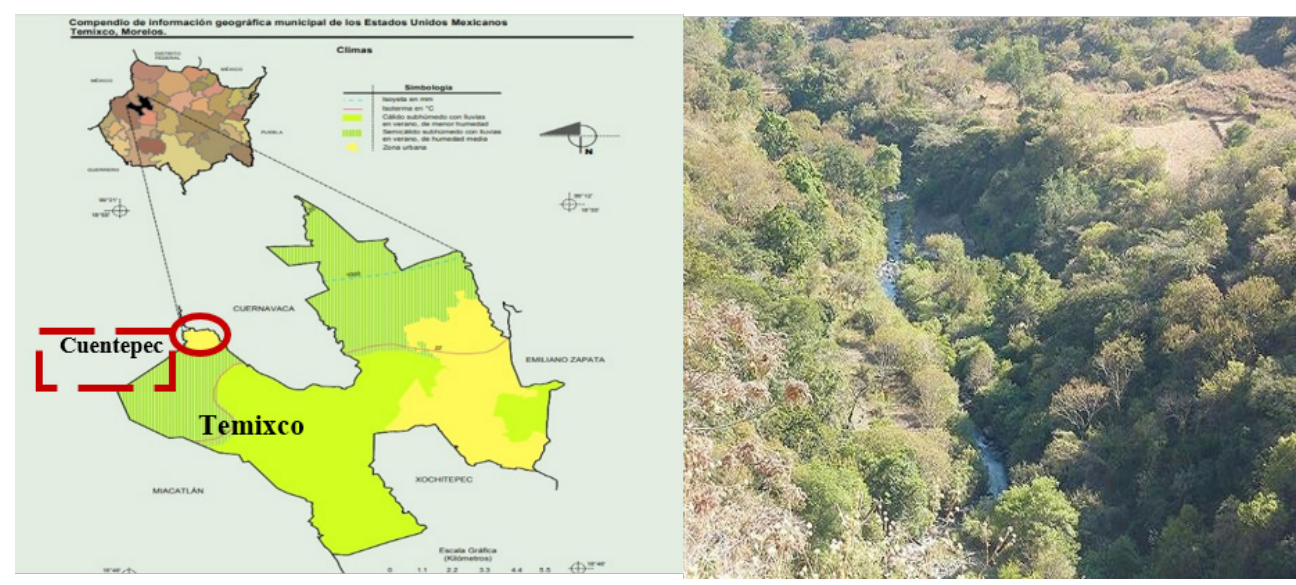

Fuente: Oxtokapan Centro Cultural, 2009

Figura 2

Etapas de crecimiento del megalóptero Corydalus sp. (ilustrativo)

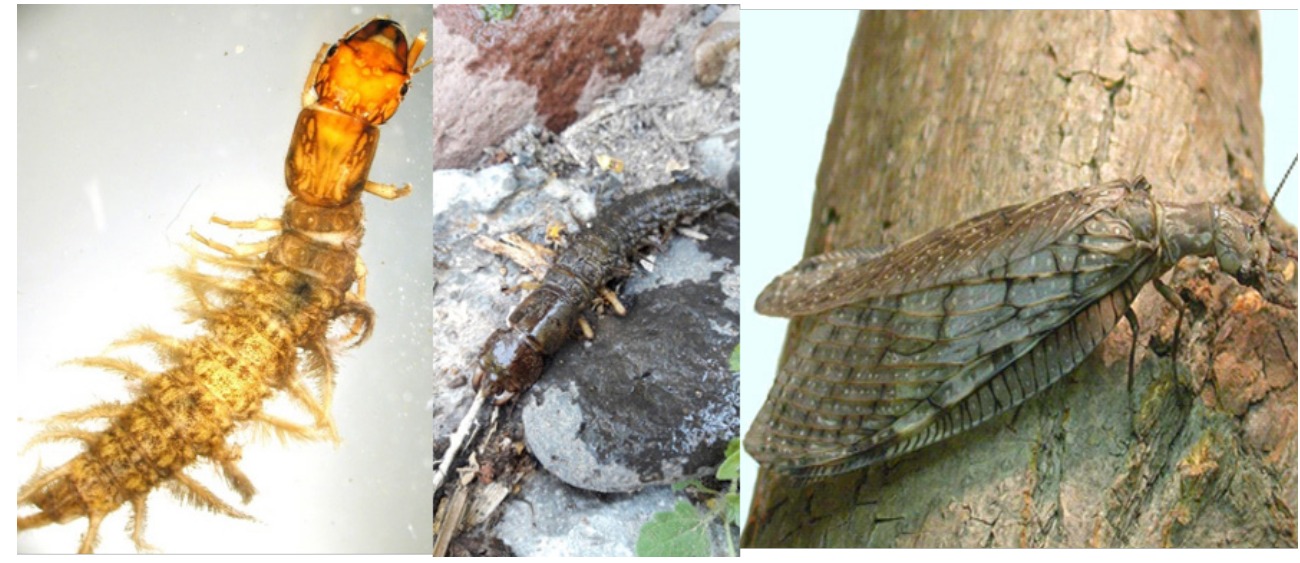

Fuente: Department of Environmental Conservation, sf; Wigney, 2006

\section{Preparación de la muestra}

Se realizó la recolección del insecto en la zona previamente descrita. Posteriormente, se limpiaron y se prosiguió a deshidratarlos en una incubadora, por cuatro días, a $37^{\circ} \mathrm{C}$, para que la humedad se fuera perdiendo gradualmente sin cambios drásticos. Para elaborar la harina, el insecto, una vez deshidratado, se pulverizó y se resguardó hasta su uso (figura 3). 


\section{Análisis proximal}

El estudio se llevó acabo en el Laboratorio de Ciencias Químicas de la Universidad Autónoma del Estado de Morelos (UAEM), de acuerdo con las normas NMX-F-068-S-1980, NMX-F-615 NORMEX-2004 y NMX-F-607 NORMEX-2002 (UNAM, 2008). Se tomaron $50 \mathrm{~g}$ de muestra (figura 3) y se prosiguió a aplicar el método Kjeldahl para la determinación del nitrógeno total. En el caso de grasa total, el análisis se realizó bajo el método de Soxhlet; por último, el análisis de hidratos de carbono se hizo mediante extracto libre de nitrógeno (Mahan \& Escott Stump, 1995).

\section{Figura 3}

\section{Preparación de la muestra para su análisis}

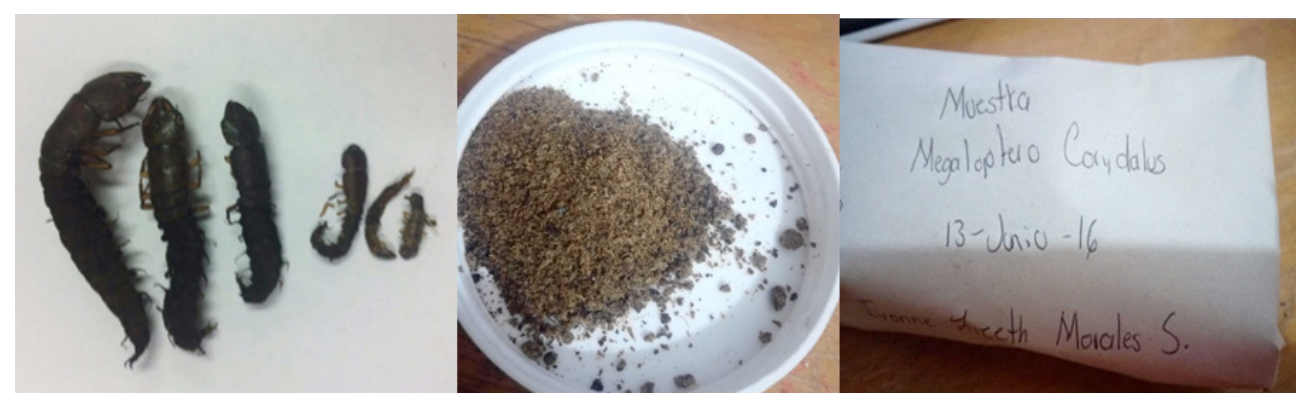

Análisis bibliográfico comparativo del valor nutritivo en varios insectos comestibles de México En relación con el valor nutritivo que poseen los insectos comestibles de México, se realizó una búsqueda bibliográfica de textos y documentos de referencia: "Edible forest insects an overlooked protein supply"; "Análisis químico y nutricional de tres insectos comestibles de interés comercial en la zona arqueológica del municipio de San Juan Teotihuacán y en Otumba, en el estado de México"; La contribución de los insectos a la seguridad alimentaria, los medios de vida y el medio ambiente; "Insectos comestibles del Estado de México y determinación de su valor nutritivo", y "Macroinvertebrados acuáticos del río Tembembe en la comunidad nahua de Cuentepec Temixco, Morelos, México". Para el análisis comparativo con el insecto comestible estudiado se revisó el libro Xopamiyolcamolli, gastronomía de bichos con muchas patas, así como Fundamentos y técnicas de análisis de alimentos.

\section{Resultados}

Los resultados obtenidos del análisis químico proximal de $100 \mathrm{~g}$ de larvas de Corydalus sp. mostraron una concentración de proteína total de $59.6 \mathrm{~g}$, que es alta en comparación con otros insectos ya registrados que se consumen en diferentes localidades de Morelos, como el jumil (43.23 g), chicatanas (46.30 g), escamol (41.68 g) y gusano de maguey (30.88 g) (gráfica 1). En este mismo análisis se observó que Corydalus sp. se acerca al valor nutricional del insecto con mayor consumo y demanda en México (el chapulín, con valor de 77.63 g) (Ló-

Año 15, núm. 37, noviembre 2019 
pez Riquelme, 2013). Aunado a estos resultados, se pudo mencionar un aporte nutrimental superior al de la carne de bovino, ya que ésta cuenta con $21 \mathrm{~g}$ de proteína en $100 \mathrm{~g}$, como se muestra en la tabla 1 (Burlingame \& Charrondiere, 2006).

\section{Gráfica 1}

\section{Contenido de proteína en $\mathrm{g}$ en diferentes insectos comestibles, integrando el que} se evaluó en este estudio

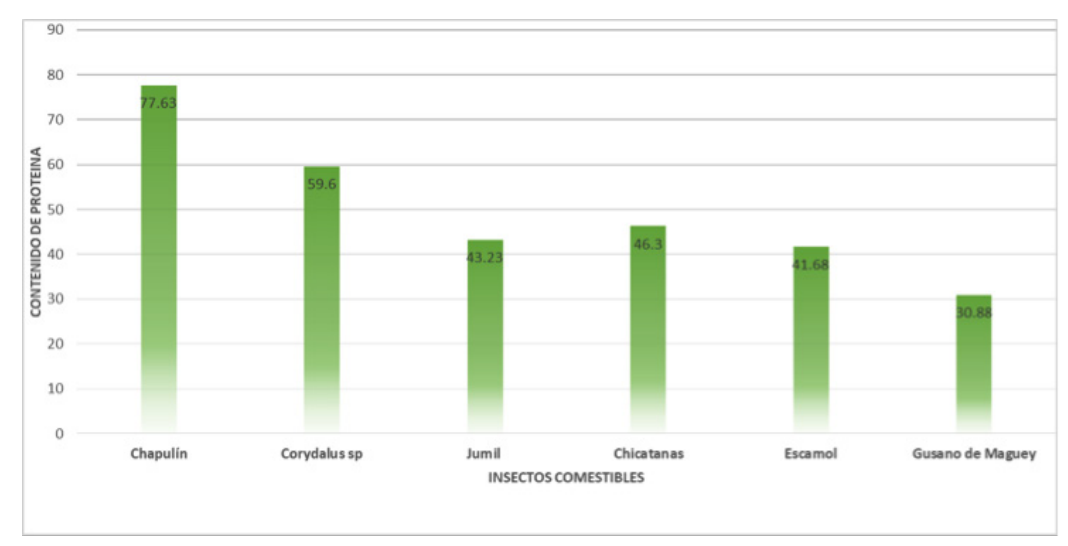

Fuente: López Riquelme, 2013

En el contenido de grasa en Corydalus sp. se pudo observar una concentración de $17.22 \mathrm{~g}$ en comparación con los insectos ya mencionados en el análisis anterior (gráfica 2). Cabe mencionar que a los insectos se les conoce por contener ácidos grasos insaturados. Por lo tanto, esta concentración se puede considerar como un ingrediente en la dieta que aporta un buen contenido de energía.

Tabla 1

Comparación del contenido proteico en gramos de Corydalus sp. con el grillo y algunos alimentos de origen animal

\begin{tabular}{llrrrrr}
\hline Especie & $\begin{array}{l}\text { Estado de } \\
\text { desarrollo }\end{array}$ & $\begin{array}{l}\text { Proteínas } \\
(\mathbf{g} / \mathbf{1 0 0} \mathbf{~})\end{array}$ & $\begin{array}{l}\text { Grasas } \\
(\mathbf{g} / \mathbf{1 0 0} \mathbf{~ g})\end{array}$ & $\begin{array}{l}\text { Sales } \\
(\mathbf{g} / \mathbf{1 0 0} \mathbf{~ g})\end{array}$ & $\begin{array}{l}\text { Fibra cruda } \\
(\mathbf{g} / \mathbf{1 0 0} \mathbf{g})\end{array}$ & $\begin{array}{l}\text { Carbohidratos } \\
(\mathbf{g} / \mathbf{1 0 0} \mathbf{g})\end{array}$ \\
\hline Chapulín & Ninfa y adulto & 77.63 & 4.22 & 2.44 & 12.3 & 4.01 \\
\hline Corydalus sp. & Prepupa y larva & 59.6 & 17.22 & - & - & 23.18 \\
\hline Pescado & - & 20.11 & 13.32 & 3.98 & 0.43 & 1.13 \\
\hline Huevo & - & 46 & 41.8 & 3.67 & 6.13 & 2.4 \\
\hline Carnederes & - & 19.4 & 25.1 & 0.9 & - & 0 \\
\hline Pollo & - & 23.4 & 4.7 & 1 & - & 0 \\
\hline Leche & - & 3.5 & 3.9 & - & - & 4.9 \\
\hline
\end{tabular}

Fuente: López Riquelme, 2013

Año 15, núm. 37, noviembre 2019 


\section{Gráfica 2}

Comparación del contenido de grasas totales en g entre los insectos que son más consumidos

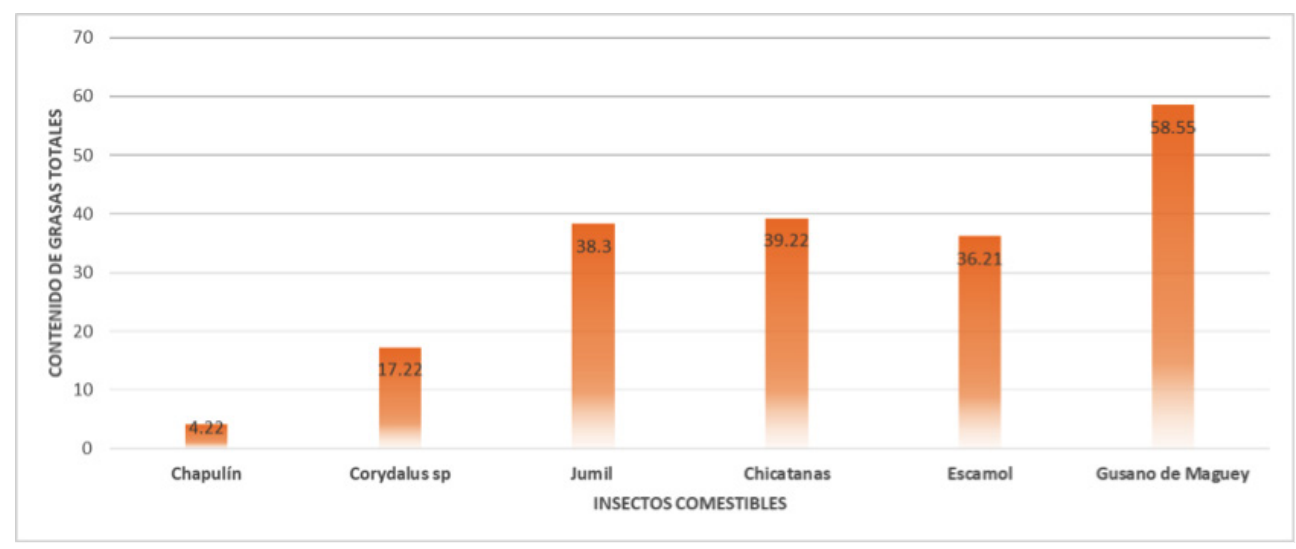

Fuente: López Riquelme, 2013

El aporte de hidratos de carbono se ve evidenciado con un valor alto en comparación con los insectos comestibles más consumidos (gráfica 3), lo cual puede deberse tanto a la cantidad de quitina como a su alimentación, así como al estado prepupal en el que se encuentra, ya que, respecto a su ciclo de vida, estos insectos tienden a almacenar macronutrientes para poder pupar y convertirse en adultos.

Gráfica 3

Contenido de hidratos de carbono

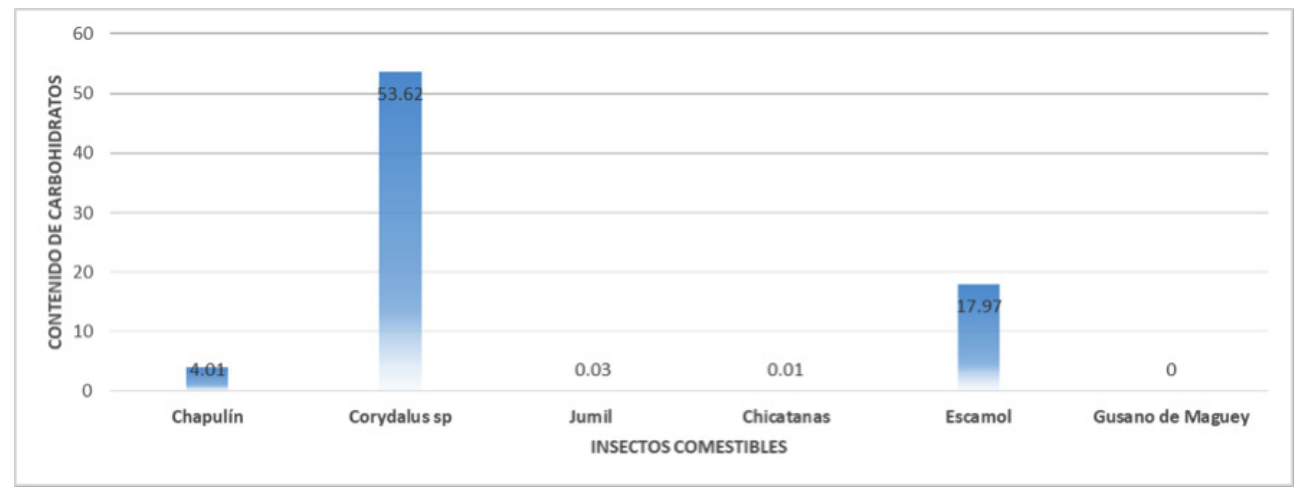

Cantidad de hidratos de carbono en $100 \mathrm{~g}$ en seco de cada uno de los insectos. El valor más alto lo contiene M. corydalus sp., en comparación con los demás insectos.

Fuente: López Riquelme, 2013

Año 15, núm. 37, noviembre 2019 
Por último, con los datos obtenidos con el análisis proximal se obtuvo también la cantidad de calorías que aporta el insecto Corydalus sp. Cuenta con un valor de $607.87 \mathrm{~g} / \mathrm{kcal}$, el cual representa un valor muy elevado y significativo, en relación con el beneficio que puede aportar al ser consumido, dadas las cantidades de macronutrientes que contiene.

\section{Discusión}

A pesar de que el aporte de proteína no fue superior al del chapulín, que es el insecto de mayor consumo en México, al compararlo con otros insectos comestibles se observó que Corydalus sp. presenta concentraciones más altas de los principales nutrimentos. Por otro lado, este estudio nos permite encontrar nuevas alternativas alimentarias, ya que esta harina, al combinarse con algún otro alimento, contaría con los valores nutrimentales que un individuo requiere en su dieta. Por lo tanto, podría contribuir en el combate a la desnutrición e inseguridad alimentaria (FAO/WHO/UNU, 1985). Sin embargo, debe garantizarse que las poblaciones del insecto no serán afectadas, ya que se debe trabajar de manera sustentable para poder aprovechar los beneficios nutricios que brinda esta especie sin afectar su ecosistema ni su supervivencia.

\section{Conclusión}

- $\quad$ El insecto Corydalus sp. presenta una concentración importante de los principales macronutrimentos.

- La harina obtenida de las pupas de Corydalus sp. es una buena alternativa alimentaria para considerarse como ingrediente tanto proteico como lipídico y con gran contenido de carbohidratos, que puede potencializar la calidad de un alimento al ser combinada.

\section{Referencias}

Burlingame, B. A. \& Charrondiere, U. R. (2006). Datos de composición de alimentos. Roma: FAO. Contreras Ramos, A. \& Rosas, M. V. (2014). Biodiversidad de Megaloptera y Raphidioptera en México. Revista Mexicana de Biodiversidad, 85, 257-263.

Department of Environmental Conservation (sf). Dobsonflies, Hellgrammites, Fishflies \& Alderflies (Megaloptera). https://www.dec.ny.gov/animals/87947.html

Domínguez, D. \& Fernández. E., H. R., (2009). Macro invertebrados bentónicos sudamericanos sistemática y biología, Buenos Aires: Fundación Migue Lillo.

FAO/WHO/UNU (1985). Necesidades de energía y proteínas, Serie de Informes Técnicos 724. Ginebra: WHO, Geneva, 2015.

Halloran, A. \& Vantomme, P. (2013). La contribución de los insectos a la seguridad alimentaria, los medios de vida y el medio ambiente. FAO, http://www.fao.org/docrep/o18/i3264s/ i3264s00.pdf

Año 15, núm. 37, noviembre 2019 
INEGI (2010). Compendio de información geográfica municipal 2010. Temixco, Morelos, Aguascalientes: INEGI.

Kourimsa, L. \& Adámkova, A. (2016). Nutritional and sensory quality of edible insects. NFS Jour$n a l, 4$, 22-26.

Kumar, R., Muzzarelli, A., Muzzarelli, C., Sashiwa. H. \& Dombi, A. J. (2004). Chitosan chemestry and pharmaceutical persectives. Chem Rev, 104, 6017-6084.

López Riquelme, G. (2013). Xopamiyolcamolli, gastronomía de bichos con muchas patas. México: Aldi de Oyarzabal.

Mahan, L. K. \& Escott Stump, S. (1995). Nutrición y dietoterapia de Krause. Pennsylvania: Interamericana McGraw Hill.

Mayorga Villalobos, A., Márquez López, Y. \& Contreras-Ramos, A. (2019). Moscas de las piedras y megalópteros (Plecoptera y Megaloptera). La biodiversidad en Morelos. Estudio de Estado 2(1).

Oxtokapan Centro Cultural (2009). Río Tembembe [foto]. https://www.flickr.com/photos/cuentepecextremo/4226718770

Ramos Elurduy, J., Pino M., J. M. \& Cuevas Correa, S. (1998). Insectos comestibles del Estado de México y determinación de su valor nutritivo. Anales Inst. Biol. Univ. Nac. Auton MexiCo, 69(1), 65-104.

Ramos Rostro, B., Quintero Salazar, B., Ramos Elorduy, J., Pino Moreno, J. M., Ángeles Campos, S. C., García Pérez, A. \& Barrera García, V. D. (2012). Análisis químico y nutricional de tres insectos comestibles de interés comercial en la zona arqueológica del municipio de San Juan Teotihuacán y en Otumba, en el estado de México. Interciencia, 37(12), 914-920. UNAM (2008). Fundamentos y técnicas de análisis de alimentos. México: UNAM.

Vantomme, P. (2010). Edible forest insects an overlooked protein supply. Unasylva, 61, 19-21.

Wigney, B. (2006). Dobsonfly [foto]. magickcanoe.com. http://magickcanoe.com/blog/?p=341

Año 15, núm. 37, noviembre 2019 